\title{
Commentary
}

\section{Where the actions of environment (nutrition), gene and protein meet: Beneficial role of fruit and vegetable juices in potentially delaying the onset of Alzheimer's disease}

\author{
Debomoy K. Lahiri* \\ Department of Psychiatry, Institute of Psychiatric Research, Indiana University School of Medicine, Indianapolis, \\ IN, USA
}

The present commentary is based on a thought provoking and experiment - driven manuscript entitled "Supplementation with apple juice attenuates presenilin -1 overexpression during dietary and genetically - induced oxidative stress" by A. Chan and T.B. Shea (University of Massachusetts Lowell, Lowell, MA) [2]. The manuscript's highlights are discussed, with a short review of the current perspective on the important topic of the effects of dietary supplementation on Alzheimer's disease (AD). The manuscript is focused to how apple juice concentrate (AJC) modulates the expression of the important $\mathrm{AD}$ - related protein presenilin (PS-1). This is a well - focused and well defined study that tests a specific hypothesis in a potentially fruitful fashion. Using an interesting animal model, the investigators in the present work have examined the effect of dietary supplementation of AJC on the overexpression of PS-1, whose variant form participates in the overproduction of amyloid $\beta$-peptide $(\mathrm{A} \beta)$, a major hallmark of AD. Notably, this work shows that AJC attenuates PS-1 overexpression during dietary and

*Address for correspondence: Debomoy K. Lahiri, Ph.D., Institute of Psychiatric Research (PR-313) Indiana University School of Medicine, 791 Union Drive, Indianapolis, IN 46202, USA. Tel.: +1 317274 2706; Fax: +1 317274 1365; E-mail: dlahiri@iupui.edu. genetically - induced oxidative stress and suggests that dietary factors are important for the oxidative stress and neurotoxic properties of $\mathrm{A} \beta$ involved in $\mathrm{AD}$ pathogenesis [2]. To expand upon the scope of the present work, it is useful to discuss some recent related work from other researchers in the field $[1,3,6,7]$.

The implication of the present work can be assessed from four important angles: i) $\mathrm{A} \beta$-mediated oxidative stress, ii) PS-1 expression, iii) nutritional and dietary factors, and iv) role of methylation. Growing evidence suggests that oxidative damage caused by $\mathrm{A} \beta$ in the pathogenesis of AD may be hydrogen peroxide mediated. Many polyphenols, the most abundant dietary antioxidants, possess stronger neuroprotection against hydrogen peroxide than do antioxidant vitamins. The premise of the work is based on studies showing that mutations in PS-1 promote AD by increasing reactive oxygen species, which is believed to be due to increased production of $\mathrm{A} \beta$. Other results suggest that folate deficiency is also associated with $\mathrm{AD}$ and potentiates the impact of both apolipoprotein E (ApoE) deficiency and $\mathrm{A} \beta$ exposure. Folate deficiency has recently been shown to increase PS-1 expression [4].

Chan and Shea tested the impact of apple juice on PS1 overexpression in vivo. Indeed, dietary supplementation with apple juice provides neuroprotection against 
ApoE deficiency, $\mathrm{A} \beta$ exposure and folate deficiency. The investigators used an appropriate and novel animal model. Their results demonstrate that dietary deficiency in folate and vitamin E increased PS-1 expression in juvenile and adult normal C57B1/6J and ApoE/- mice and in aged normal mice. Notably, supplementation with apple AJC attenuated or prevented these increases. What would be the mechanism of such overexpression of the PS-1 gene? This leads to the important methylation portion of the studies. Previous studies indicate that deficiency in S-adenosylmethionine (SAM) causes impaired DNA methylation [8]. How is this deficiency brought in? That leads to the nutrition part of the story. Indeed, SAM is rapidly depleted following folate deprivation (from the diet) [7]. Thus, reduced folate level results in lower SAM which in turn causes hypomethylation leading to PS-1 overexpression. So not surprisingly, direct supplementation with SAM (hypermethylation) would attenuate PS-1 overexpression.

Two important results emerge from this work. First, AJC contained SAM levels comparable to those capable of suppressing PS-1 overexpression. This may suggest that the SAM content of AJC constitutes a potential mechanism to arrest PS-1 overexpression. Second, AJC may be able to provide neuroprotection by mechanisms in addition to its antioxidant potential

Some recent articles and data related to this work are worth mentioning. In a complementary study of the present kind, it has been shown that nearly 2,000 Japanese Americans, individuals who regularly consumed fruit and vegetable juices had a significantly lower risk for developing AD [3]. They tested whether consumption of fruit and vegetable juices, containing a high concentration of polyphenols, decreases the risk of incident probable AD in the Kame Project cohort, a population - based prospective study of Japanese Americans in King County, Washington, who were dementia - free at baseline (1992-1994) and were followed through 2001. Thus, these results suggest that fruit and vegetable juices may play an important role in delaying the onset of $\mathrm{AD}$, particularly among those who are at high risk for the disease.

Regarding generality of the mechanism, the authors rightly pointed out that there are multiple pathways for the neuroprotective function of AJC [2]. In addition to its well know antioxidative properties, the effect on methylation has recently been gaining momentum. As the present work showed, increased methylation by the dietary supplementation of SAM could down regulate PS1 expression [2]. However, the exact mechanism of this phenomenon is far from clear. For example, is the effect of AJC on gene expression a transient phenomenon at the latter part of life (adult/old stage), an accumulated effect over longer period of time (early through adult life), or developmental (fetal/early) with a delayed expression? Work has been carried out to understand the "timing" of such an effect [5,6]. The interactions between environmental factors, such as diet, and gene have been well documented in several disorders [1]. The classic "black-box" model of "gene environment interaction" is worth considering in light of the recently proposed "LEARn" model (Latent Early-life Associated Regulation) [6]. The LEARn model postulates latent modulation of specific gene's expression, triggered at the developmental stage. According to this model, environmental agents, such as heavy metals and toxins, and dietary factors, such as folate and cholesterol, could perturb gene regulation in a long - term fashion, beginning at early developmental stages, but that these perturbations do not have pathological results until significantly later in life. This model operates on the regulatory region (promoter) of the gene and by the effect of methylation at certain specific sites within the promoter of specific genes. In essence it addresses the imposition of "somatic epitypes" on underlying gene primary DNA sequence [5]. The somatic epitype would govern gene expression levels and directly give rise to the specific phenotype.

Overall, the authors present interesting data on the effect of dietary supplementation with apple juice concentrate on PS-1 expression. The subject matter is interesting with regard to gene regulation research in general as well as AD research in particular. Although it may be still debatable when and how the actions of environment, nutrition, gene and protein converge, it is ample clear from aforementioned animal, human, epidemiological and genetic studies that there is beneficial role of fruit and vegetable juices in delaying the onset of Alzheimer's disease. These results may lead to a new avenue of inquiry in the prevention of AD.

\section{Acknowledgments}

This work was supported in part by grants from Alzheimer's Associations (Zenith award) and the National Institutes of Health (AG18379 and AG18884). Sincere thanks to B. Maloney and J. Bailey for their useful comments.

Conflict of Interest Statement: The author declares that he has no competing financial interests. 


\section{References}

[1] A. Caspi and T.E. Moffitt, Gene-environment interactions in psychiatry: joining forces with neuroscience, Nat Rev Neurosci 7 (2006), 583-590.

[2] A. Chan and T.B. Shea, Supplementation with apple juice attenuates presenilin-1 overexpression during dietary and genetically-induced oxidative stress, J. Alzheimers Dis 10(4) (2006), 353-358.

[3] Q. Dai, A.R. Borenstein, Y. Wu, J.C. Jackson and E.B. Larson, Fruit and vegetable juices and Alzheimer's disease: the Kame Project, Am J Med 119 (2006), 751-759.

[4] A. Fuso, L. Seminara, R.A. Cavallaro, F. D'Anselmi and S. Scarpa, S-adenosylmethionine/homocysteine cycle alterations modify DNA methylation status with consequent deregulation of PS1 and BACE and beta-amyloid production, $\mathrm{Mol} \mathrm{Cell} \mathrm{Neu-}$ rosci 28 (2005), 195-204.
[5] D.K. Lahiri and B. Maloney, Genes are not our destiny: the somatic epitype bridges between the genotype and the phenotype, Nature Rev Neurosci (2006), in press.

[6] D.K. Lahiri, B. Maloney, M.R. Basha, Y.W. Ge and N.H. Zawia, How and when environmental agents and dietary factors affect the course of Alzheimer's disease: the "LEARn" model (Latent Early-life Associated Regulation) may explain the triggering of AD, Curr Alzheimer Res (2007), in press.

[7] M.D. Niculescu and S.H. Zeisel, Diet, methyl donors and DNA methylation: interactions between dietary folate, methionine and choline, J Nutr 132 (2002), 2333S-2335S.

[8] C.J. Roberts and E.U. Selker, Mutations affecting the biosynthesis of S-adenosylmethionine cause reduction of DNA methylation in Neurospora crassa, Nucleic Acids Res 23 (1995), 48184826. 